\title{
Two Examples of Nursing Working System in Emergency Medical Services
}

\author{
Öznur Kavaklı \\ Gülhane Military Medical Academy, School of Nursing, Ankara, Turkey
}

\begin{abstract}
A nurse who is an indispensable part of the emergency service team should also be aware of her duties, authorities and responsibilities, and skills required for her profession. In this report, the duties and responsibilities of nurses in two different systems have been presented, discussed and compared. (Eurasian J Emerg Med 2016; 15: 44-7)
\end{abstract}

Keywords: Nursing, emergency medicine, working system

\section{Introduction}

Emergency medical service is a special field within healthcare. Emergency units are not only the busiest, most stressful, and complex sections in health institutions but also are the ones where saving lives is a priority, patients requiring attention are evaluated, and treatment and care are provided.

Besides having more distinctive properties and undergoing special training than those in other units, the specification of the nurses' duties, authorities, and expanding roles is essential for the effective management of emergency patients' care and their satisfaction (1). Working long and volatile hours, emergency nurses fall into a risky group in which quick diagnosis and treatment are required. There are patients with malignant diseases and their relatives, forensic cases involved, deaths are commonly encountered, a number of medical devices are being employed (2). The expansion of authority and implementing roles of emergency nurses relieve the pressure in emergency departments, decreasing the waiting time for the patients (3).

In this observational report, topics about nursing services, organization in emergency services, roles, and training have been observed and discussed.

\section{First Example of Emergency Units and Emergency Nursing Services}

The structure of an emergency unit is like an arena and is modular in shape. For trauma patients, a special trauma unit is carefully appointed. In this organization, emergency nurses are a part of trauma nurses.

\author{
a) Departments Assigned to Emergency Nurses: \\ - Triage \\ - Units of emergency department A, B, C, D \\ - Pediatric emergency
}

\section{b) Hierarchical Structure of Emergency Nurses and their Training}

Emergency nurses include charge and training nurses. During the night shift of the charge nurse and assistant and staff nurses, the charge nurse supervises all the work.

It is suggested by the Emergency Nurses Association (ENA) that the certificates given in Table 1 be available. Emergency nurses are required to obtain these certificates and are obliged to renew their certificates at certain intervals (2-4 years). All emergency nurses treating trauma patients have the obligation to obtain the Trauma Nursing Core Course (TNCC) certificate.

In the blue code warning system, emergency nurses are involved in immediate responses in cases of emergency inside the hospital (e.g., to help a patient with syncope in the corridor). They, however, are not a part of the Rapid Response Team. These units have been assigned for quick and urgent responses within the clinic.

\section{c) Duties of Charge Nurses}

A charge nurse is the person who is willing to work, has 5 years of experience in emergency services, and possesses all the certificates that emergency nurses are obliged to have; moreover, they are selected by the supervisor and emergency nurses, works in shifts, and is the head of the nurses. A charge nurse is stationed somewhere close to the emergency ambulance entrance. They sit at a desk equipped with a large computer with its monitor and an ambulance

Correspondence to: Öznur Kavaklı e-mail: okavakli@gata.edu.tr

Received: 12.04.2015 Accepted: 19.10.2015

(C) Copyright 2016 by Emergency Physicians Association of Turkey - Available online at www.eajem.com DOI: 10.5152/eajem.2016.78557 
call device. She receives the first piece of information about the patients (including pediatrics and adults cases) and is responsible for welcoming them, placing them in the patients' module, and making all the decisions. She also organizes the emergency unit and prepares the trauma rooms for good use when on call. She is responsible to emergency charge nurse. While working, she does not provide any patient care service.

During the transfer of patients with the "red" trauma code to the emergency unit, a charge nurse gets the first piece of information. When she receives the red code, she makes sure the entire trauma team gets into action in 2 minutes.

d) Triage: In emergency departments, there are two separate lounges, namely general and triage. The triage nurse receives the story as to the patient's problem and gives the triage code. “Emergency Severity Index' is employed in this service. Triage nurses decide on the appropriate care protocol, thus saving time. It has been reported by the Emergency Nurses Association (ENA) that a triage nurse is required to work at least for 6 months in an emergency unit in order to have strong communication skills and have the ability to work independently and to make quick decisions. A sample application for the triage care protocol is shown in Table 2.

e) Emergency Nursing Applications: An emergency nurse is responsible for five modules and serves five patients at once. After a brief introduction, the emergency nurse gathers information about the patient's story. Before any attempt she makes, she takes spoken and written orders and makes sure they match on the hospital register system and the information form filled out in pen. There is a computer system in which data is immediately recorded and patients can be monitored at any time at the emergency nurse desk. Nurse call buttons are actively used in the emergency patient modules. Within the hospital information system, nurse register headings are as follows:

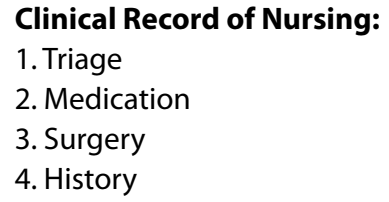

Table 1. Emergency nurse certificate programs

\begin{tabular}{|l|l|}
\hline 1. & Certified Emergency Nurse \\
\hline 2. & Trauma Nursing Core Curriculum \\
\hline 3. & Emergency Nursing Pediatric Course \\
\hline 4. & Basic Life Support \\
\hline 5. & Advance Cardiac Life Support \\
\hline 6. & Pediatric Advance Life Support \\
\hline
\end{tabular}
5. Allergies
6. Interventions
7. Physical Assessment
8. Nursing Progress Notes

Positions and Roles of the Trauma Team

The positions and roles of the trauma team have been summarized in Figure 1.

The duties of the emergency nurses in the trauma team:

\section{1) Primary duties of the nurse:}

- Prepares the trauma room

- Keeps a record of all of the resuscitation data and applications

- Assumes the patient's care after the post-trauma.

\section{2) Secondary duties of the nurse:}

- Makes sure the IV vein passage is open

- Manages laboratory tests without being warned by the trauma team members

- Provides fluid, blood, and medicine infusion

- Applies NG, OG, and Foley catheter if necessary

- Organizes the trauma room

Each patient admitted to the emergency unit is given care by more than one nurse. One reason for this is that there is an insufficient number of emergency nurses and another is that are too many patients coming.

Some of the emergency nurses have a "Emergency Nursing Certificate" approved by the Ministry of Health. An emergency nurse receive in-service training and clinic training sessions once a week. In addition to various scientific studies, they attend scientific activities and conventions organized by Emergency Medicine Associate, Emergency Medicine Specialist Associate and Emergency Nursing Associate.

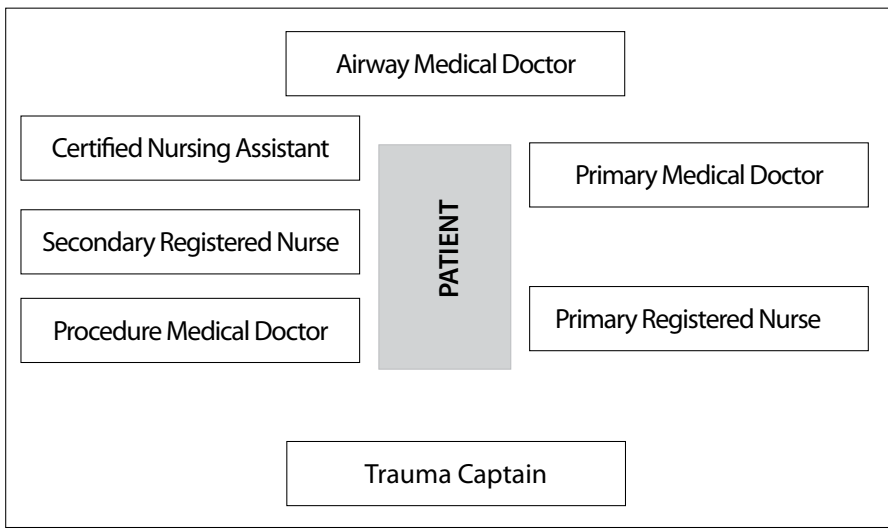

Figure 1. Position and roles of trauma team

Table 2. Adult emergency triage care protocol (for patients aged 18 and over)

\begin{tabular}{|c|c|c|c|c|}
\hline Patient complaint & Nursing applications & Laboratory & Drug therapy & Radiological examinations \\
\hline $\begin{array}{l}\text { Chest pain associated } \\
\text { with coronary artery } \\
\text { disease }\end{array}$ & $\begin{array}{l}\text { Cardiac monitorization, } \\
\text { oxygen saturation, } \\
\text { electrocardiogram (ECG); } \\
\text { start with } 2 \text { It/min through } \\
\text { nasal cannula, open the } \\
\text { suitable IV way }\end{array}$ & $\begin{array}{l}\mathrm{Na}, \mathrm{K}, \mathrm{Cl}, \mathrm{paCO}_{2^{\prime}} \mathrm{paO}_{2^{\prime}} \\
\mathrm{BUN}, \mathrm{creatinine} \text { glucose, } \\
\text { PT, INR, CK, CK-MB, trop, } \\
\text { 12-lead ECG }\end{array}$ & $\begin{array}{l}0.4 \mathrm{mg} \\
\text { nitroglycerine } \\
5 \mathrm{~min} \times 3 \text { doses }\end{array}$ & (Chest X-ray) \\
\hline
\end{tabular}




\section{Second Example Emergency Unit and Emergency Nursing} Services

The emergency service is a step 3 healthcare service and is an arena and modular system. It has three trauma rooms prepared for trauma patients. However, there is not a planned and appointed trauma team.

\section{a) Units where emergency nurses are employed: \\ - Rapid care \\ - Arena}

\section{b) Working system and training of emergency nurses}

In the emergency arena unit, all the clinical operations and emergency nursing work system are monitored by the most senior nurse appointed by the emergency nurse. There is also a graduate training nurse who works with emergency nurse.

Emergency nurses are not employed in the triage. In the rapid care unit which is an extended part of emergency triage, injection, taking samples for laboratory tests, opening IV vein lines, and ECG can be applied. When there are sick and wounded people because of disasters or terrorist attacks, emergency nurses are given additional duties to watch services. They are responsible for the availability of medical devices and equipment in the emergency arena. In the arena, emergency nurses act according to verbal or written orders, enter manually the nurse part of the emergency patient form used in association with doctors and nurses into the hospital information system.

\section{Discussion}

The population growth and the burden of chronic diseases at the present have increased the applications to emergency services. Owing to increased pressure of the services in emergency units, the adoption of new service models seems inevitable (3). In the first emergency service we have observed, a nurse is responsible for five modules and is responsible for treatment of five patients. In fact, she is responsible for the primary treatment of the five patients she observes, immediate treatment and their procedures. In the second emergency service, however, primary nursing applications are employed and at other times, the other emergency patients' procedures are handled. Though not planned, in critical cases, three or four nurses deal with a single patient at the same time. The service organization of the emergency nurses and a careful planning of the work force play an important part in the provision of high quality emergency care services.

Emergency services are the front door of the patient care services. All applications at the emergency services, registration and first nursing evaluation are carried out on the stretcher. Immediate use of the stretcher speeds up the process of acceptance to the emergency services which also includes triage period. Emergency nurses should be responsible for the safety, continuity of care and effective simplification of patient flow (4). In the first emergency service, as individuals pay for their own healthcare, the flow is relatively slower. In the second emergency department, on the other hand, the number of patients flow is higher and a single nurse has to treat more than one patient at once. In both of the emergency departments, the dynamic actions of the emergency nurses facilitate the patient flow.

Opinions of the emergency nurses from Israel have been taken about the notification of the patients and triage. It has been reported that new and broad authorities and increase in practical roles of the nurses will not only decrease the pressure in emergency department but also shortens the waiting period of the patients. Most of the participants in the group asked for authorization and tasks in the triage (5). In the first emergency department observed, the triage nurse is authorized to make a decision in accord with the patients' triage code. All the requests about the emergency patient are given at the outset by the triage nurse, thus saving time. In the other emergency department observed, emergency nurses were not assigned in triage, but they seem to have been employed in the minor care unit.

In this dynamic environment, the effective role distribution of the health professionals in emergency services, planning of the building and working system and its continuation are instrumental in overcoming the problems in the information flow. It has been thought that, in order to enhance the quality of the patient care, an emergency team should focus on the interdisciplinary care and the role distribution between nursing and medical jobs should be re-designed. In emergency departments, such matters as technology-based communication system and simultaneous interactions with doctors contribute to the role expansion of the nurse. Cognitive work distribution pattern increases collaboration (6). In the first emergency department observed, the charge nurse can be given as an example for the role expansion of an emergency nurse. Through the technology-based communication system and simultaneous interactions with the doctors, a charge nurse speeds up the transportation of a patient to the emergency treatment and increases the survival chance of the patient. Also, she acts as a coach by providing communication and collaboration among emergency crew. In emergency departments, the presence of emergency nurses experienced, competent and trained as permanent members of the emergency personnel enables a comprehensive evaluation of the patients and the smooth improvement of management skills. In the second emergency department, this task is partly fulfilled by a senior nurse though not formal.

In order to offer better services to patients, emergency nurses should regulate their services with better care and in a more planned way. According to a study, the time that emergency nurse spends with a patient supports the idea of holistic healthcare. Also it is reported that continuous training and further research on the specific patient groups visiting emergency departments (such as pediatrics, mental health, and the aged) are required (7). In the first emergency department, the obligation of obtaining some of the certificates mentioned above and their renewal (2-4 years) will increase the quality of the services. In the second emergency department, there is no such requirement. Besides experience, having received the required education level and attaining clinical approval are among the musts for emergency nurses. In our country, though the importance of certificate programs is being better understood, legal and institutional obligations should be clearly expressed. 


\section{Conclusion}

In advanced countries, nurses can provide efficient services mainly because of the standardization of the services. Institutional standardization includes nursing services too. The application standardization of emergency nurses who work together with the emergency team and the service organization and planning of the workforce play an important role in the provision of quality healthcare services.

In this review, two distinct nursing applications in emergency departments have been analyzed. In both these departments, the dynamic actions of emergency nurses make the management of the patient flow easier during patient registration and evaluation. The expansion of roles decreases the waiting period of the patients. Clinical authorization of the emergency nurses will increase team dynamics and collaboration.

Peer-review: Externally peer-reviewed.

Conflict of Interest: No conflict of interest was declared by the author.
Financial Disclosure: The author declared that this study has received no financial support.

\section{References}

1. Isır AB. Duties, Legal and Ethical Responsibilities of the Emergency Nurses. Turk J Emerg Med 2006; 6: 90-6.

2. Kebapçı A, Akyolcu N. The impact of working environment on fatigue levels for Emergency Nurses. Turk J Emerg Med 2011; 11: 59-67.

3. Jennings N, Clifford S, Fox AR, O'Connell J, Gardner G. The impact of nurse practitioner services on cost, quality of care, satisfaction and waiting times in the emergency department: A systematic review. Int J Nurs Stud 2015; 52: 421-35. [CrossRef]

4. Scrofine S, Fitzsimons V. Emergency department throughput strategies for success. JONA 2014; 44: 375-7. [CrossRef]

5. Azuri P, Haron Y, Riba S. Israeli emergency department nurses' attitudes to an extension of their role and powers. J Clin Nurs 2014; 23: 261-7. [CrossRef]

6. Gilardi S, Guglielmetti C, Pravettoni G. Interprofessional team dynamics and information flow management in emergency departments. J Adv Nurs 2014; 70: 1299-309. [CrossRef]

7. Dunnion ME, Griffin M. Care planning in the emergency department. Int Emerg Nurs 2010; 18: 67-75. [CrossRef] 